\title{
Prevalence of HIV, Hepatitis B, Hepatitis C and Syphilis Infections among Patients in a Psychiatric Hospital in Ghana
}

\section{Benedict Osei-Tawiah ${ }^{1}$, Theophilus B Kwofie ${ }^{1}$, Mohamed Mutocheluh', Richard Ephaim ${ }^{2}$, and Emmanuel Awusah Blay ${ }^{3 *}$}

${ }^{1}$ Department of Clinical Microbiology, School of Medical Sciences, Kwame Nkrumah University of Science and Technology, Ghana

${ }^{2}$ Department of Medical Laboratory Technology, University of Cape Coast, Ghana

${ }^{3}$ Graduate School of Medical and Dental Sciences, Tokyo Medical and Dental University, Japan

*Corresponding author: Emmanuel Awusah Blay, Graduate School of Medical and Dental Sciences, Tokyo Medical and Dental University, 1-5-45 Yushima, Bunkyo-ku, 113-8519, Tokyo, Japan

\begin{abstract}
Background: In developing countries like Ghana, people with serious psychiatric disorders do not have equal access to health care as normal non-mentally challenged people do. Moreover, mental health care generally focuses on physical health screening, health education, and improving access to treatment in primary and secondary care to the detriment of sexual health needs including prevention of sexually transmitted infections. Studies have shown the risk of blood-borne virus infections such as HIV, hepatitis B virus, and hepatitis $C$ virus has increased in the last few decades among people with psychiatric disorders. This study sought to determine the prevalence of HIV, HCV, HBV and syphilis infections in patients with psychiatric disorders.
\end{abstract}

Methods: In a cross-sectional study, 200 inpatients of Ankaful psychiatric hospital, aged $\geq 18$ years diagnosed with schizophrenia, bipolar disorder, depression, psychiatric disorders, and substance abuse and dependence disorders formed the study population. Questionnaire interviews were conducted to obtain personal information and sociodemographic characteristics. Serological tests were performed on serum and plasma samples of participants to determine the prevalence of HIV, HCV, HBV and syphilis.

Results: Seroprevalence for HIV was 5\% (10/200), HBV was $9.5 \%(19 / 200)$, syphilis was $13.5 \%(27 / 200)$ and HCV was $2 \%(4 / 200)$. Results indicated a significant association between Schizophrenia diagnosis and the seropositivity for anti-HIV antibodies, anti-HBV antibodies and anti-T. pallidum antibodies. Co-infections and multiple infections among study participants showed $1.5 \%(3 / 200)$ seroprevalence of HBV and HIV co-infections. HBV and syphilis co-infections was $1.0 \%(2 / 200)$ and HIV and Syphilis co-infection was
$0.5 \%(1 / 200)$. HCV and syphilis co-infection was also $0.5 \%$ $(1 / 200)$. One patient had HIV, HBV and Syphilis $0.5 \%$ $(1 / 200)$. The majority of the study subjects who tested positive for HBV, HCV and syphilis had no history of sex trafficking.

Conclusion: The current study shows that people with psychiatric disorders in Ghana are at increased risk of blood-borne viruses and STIs compared to the general population. Our results could form the baseline data for decision making by the health authorities, as there is an urgent need for developing prevention and care strategies for psychiatric patients in Ghana.

\section{Keywords}

Psychiatric disorders, HIV, Hepatitis B, Hepatitis C, Syphilis, seroprevalence, Psychiatric hospital, Ghana

\section{Introduction}

Reports by the World Health Organization (WHO) indicate that approximately 2.2 million Ghanaians suffer from mental disorders, and 650,000 of that suffer from severe mental disorder [1]. These include mental disorders such as schizophrenia, schizoaffective disorder, bipolar disorder, or psychosis. Notwithstanding the significant number and importance of psychiatric healthcare, there exists a huge deficit in terms of treatment and access to healthcare [2]. Current estimates predict about $98 \%$ treatment gap i.e. persons who need treatment but do not have access to adequate treatment.

Citation: Osei-Tawiah B, Kwofie TB, Mutocheluh M, Ephaim R, Blay EA (2019) Prevalence of HIV, Hepatitis B, Hepatitis C and Syphilis Infections among Patients in a Psychiatric Hospital in Ghana. Int J Virol AIDS 6:050. doi.org/10.23937/2469-567X/1510050

Accepted: March 16, 2019: Published: March 18, 2019

Copyright: (c) 2019 Osei-Tawiah B, et al. This is an open-access article distributed under the terms of the Creative Commons Attribution License, which permits unrestricted use, distribution, and reproduction in any medium, provided the original author and source are credited. 
In Ghana, mental health care generally focuses on physical health screening, health education, and improving access to treatment in primary and secondary health centers [3]. Sexual health needs of psychiatric patients receive little or no attention thus increasing the risk of psychiatric patients to sexually transmitted infections. While no representative epidemiological studies have been conducted to calculate the prevalence of HIV and other sexually transmitted diseases among people with different types of mental illness, a number of studies utilizing secondary data in different parts of sub-Saharan Africa have found high rates of HIV and sexually transmitted diseases (STDs) among people who were treated in specialty mental health settings [4].

Studies have shown the risk of blood-borne virus infections such as Human Immune-deficiency virus (HIV), hepatitis B virus (HBV), and hepatitis C virus (HCV) has significantly escalated in the last few decades among people with psychiatric disorders [5]. The most common of these psychiatric disorders include major depressive disorders, dementia, substance use disorders, posttraumatic stress disorders (PTSDs), childhood conduct disorders, and cognitive and generalized anxiety disorders [6]. A major cause for this increased risk is due to the association of psychiatric disorders and substance abuse [7]. Substance use disorders are a critical issue among psychiatric patients, as they are not only risk factors for HIV and other viral transmissions but are also factors associated with reduced antiretroviral therapy adherence, clinical progression, reduced virological response and mortality [8]. Viral diseases such as HIV, $\mathrm{HBV}$ and $\mathrm{HCV}$ are transmitted by parenteral contact with contaminated body fluids (blood and blood products; contaminated instruments and needles; semen and vaginal fluids) and through unprotected sex (anal, vaginal, or oral) or by congenital transmission from mother to baby [9].

In Ghana, approximately $17 \%$ of HIV-infected individuals are also chronically infected with hepatitis $B$ virus [10]. In direct contrast to developed countries, most HBV infections are acquired during early childhood, so patients with HIV/HBV co-infection have usually had chronic HBV infection for many years by the time they present with (or are tested for) HIV infection [11]. Although there is currently a national control strategy and action plan for HIV, there is none for the prevention and control of HBV, HCV and Syphilis in Ghana. Current STI control efforts usually target symptomatic patients and have failed to identify individuals with psychiatric disorders until serious complications develop.

This research thus sought to determine the prevalence of HIV, HCV, HBV and syphilis infections in patients with psychiatric disorders. Additionally, we analyzed the related risk factors associated with STI prevalence in psychiatric patients. The objective of this research is to provide comprehensive data to aid in policy decisions in providing a national control plan for psychiatric healthcare in Ghana.

\section{Methods}

\section{Study design and setting}

A hospital based cross-sectional study was conducted at Ankaful psychiatric hospital in Central Region, Ghana. The hospital functions as one of the three (3) psychiatric hospitals in the country with additional responsibilities as a teaching hospital for both medical students and nurses in mental health and psychiatry. Persons aged 18 years and above, diagnosed with schizophrenia, bipolar disorder, depression, psychiatric disorders, substance abuse and dependence disorders admitted as in-patients at the hospital were considered eligible for the study. A well-structured questionnaire was used to obtain information on background characteristics, knowledge about HIV, HBV, HCV, and syphilis and vaccination. Information on history about forced sex and sex work, substance use/abuse behavior of all participants was collected. Confidentiality was assured at all times with the questionnaire given unique alphanumeric identifiers.

\section{Study population and sample size}

All in-patients of Ankaful psychiatric hospital, aged $\geq 18$ years diagnosed with schizophrenia, bipolar disorder, depression, psychiatric disorders, and substance abuse and dependence disorders formed the study population. For calculation of the sample size, we assumed a minimum of $10 \%$ and a maximum $90 \%$ for STI sero-prevalence as expected frequency of the factor under study and a confidence level of $95 \%$. The result of the calculation was a minimum of 138 subjects for accurate statistical inference [12]. A convenience sampling method was used to sample for the participants and elements of bias were controlled by ensuring that the sample was a miniature of the whole population under study. A total of 246 patients were initially recruited for the study out of which 233 were passed as psychologically stable by the Medical Officerin-charge to partake in the study. Thirty-three (33) persons representing $14.2 \%$ of total eligible persons did not give consent to be part of the study and were duly excluded.

\section{Ethical considerations}

The Committee on Human Research, Publications and Ethics of the Kwame Nkrumah University of Science \& Technology (CHPRE/KNUST) and the ethics board of the Ankaful Psychiatric hospital respectively, approved the study protocol. Written informed consent was also obtained from the participants before enrollment into the study. Emphasis was placed on the fact that participation was voluntary and there was the liberty of withdrawing from the study at any time without further obligation. 
All patients who tested positive for HIV, HCV, HBV and syphilis were referred to the resident medical doctor at Ankaful Psychiatric Hospital for the appropriate disease treatment and management.

\section{Socio-demographic and STI risk related data collection}

Close-ended questionnaires were administered to collect demographic as well as STI-related risk factors data. Questionnaires were administered in an interview format in participants' mother language for easy understanding. Questions sought personal information on participants including their knowledge about various STIs and related infection transmission risk factors. The possible infection transmission risk factors considered in the questionnaire included sexual orientation and sexual preferences, multiple sexual partners, substance abuse and condom usage.

\section{Blood sample collection}

From the cubital veins of participants, approximately 3 $\mathrm{ml}$ of blood was drawn using a sterile butterfly hypodermic syringe and needles and dispensed into a serum separator tube and transported on ice to the laboratory. The samples were centrifuged at $1500 \mathrm{rpm}$ for 10 minutes to obtain serum and stored at $-80{ }^{\circ} \mathrm{C}$ until analyzed.

\section{Serological diagnosis of HIV-1/2}

Serum obtained from the study subjects were screened using two commercial test kits recommended for use nationwide by the Ministry of Health in Ghana. Antibodies to HIV were detected using Ora Quick ADVANCE Rapid HIV-1/2 antibody (first line screening) and the positive test results were confirmed with the One Step Anti-HIV (1 and 2) Tri-line Test. In the event of a discordant results between the two test kits, a commercially available Western Blot Assay kit (HIV BLOT 2.2) supplied by MP Diagnostics was used to decide the results. The One Step Anti-HIV (1 and 2) Tri-line Test is a colloidal gold enhanced rapid immuno-chromatographic assay for detecting antibodies of all isotypes (IgG, IgM, IgA) to HIV 1 and HIV-2. All reactive samples from the One Step Anti-HIV1 and 2 Tri-line Test (First Response) were retested with OraQuick $^{\circledast}$ ADVANCE Rapid HIV1/2 Antibody Test (OraSure Technologies, Inc. USA) for confirmation. All tests were performed strictly according to the manufacturer's instructions.

\section{Serological diagnosis of hepatitis B virus}

Serum samples from the study subjects were tested for hepatitis B surface antigens ( $\mathrm{HBsAg}$ ) using the Accu-Tell ${ }^{\circledR}$ One Step HBsAg rapid test (AccuBio Tech Co., Ltd.). The Accu-Tell ${ }^{\circledR}$ One Step HBsAg rapid test employs sandwich immunoassays in the detection of $\mathrm{HBsAg}$ in serum or plasma. Antigens in sample bind to recombinant anti-HBsAg antibodies conjugated to colloidal gold particles in the sample well and resulting mixture move alone the cellulose membrane which contains the test region and the control region. The $\mathrm{HBsAg-HBs}$ antibody-conjugate complex binds to the anti-HBsAg antibodies immobilized in the test region and a color develops which indicates positive results. Color development at both test and control region indicates valid positive test results and color development at the control region alone is an indication of valid negative test results. All positive samples were confirmed using Roche COBAS e411 analyzer with elecsys HBsAg II quant test (Roche Diagnostics, Germany).

\section{Serological diagnosis of Hepatitis $\mathbf{C}$ virus}

Serum samples from the study subjects were tested for hepatitis C virus (HCV) using Rapid Test kits (Cortez Diagnostics, California USA). The test kit operates on the principle of double antigen sandwich immunoassay. Purified recombinant antigens identify anti-HCV antibodies specifically. Negative test results show only one colored band on the control $\mathrm{C}$ region with no apparent band on the test $(T)$ region. Positive HCV Rapid test results show a pink-colored control $\mathrm{C}$ band, with a distinct pink-coloured band in the test $(\mathrm{T})$ region.

\section{Syphilis serological tests and experimental proce- dures}

Samples were screened for syphilis using AccuTell ${ }^{\circledR}$ One Step Anti-Treponema pallidum test (AccuBio Tech Co., Ltd.). This rapid test employs the sandwich principle in detecting antibodies to Treponema pallidum in serum. The test card has a sample well in which Treponema pallidum antigen (TP Ag 1) conjugated with colloidal gold particles has been immobilized. Recombinant Treponema pallidum antigens (TP Ag 2) has been immobilized in the test region and antiTreponema pallidum antibodies in the control region. On application of the test sample, the anti-Treponema pallidum antibodies bind to the conjugated TP Ag 1 which forms a colored mixture. As the colored mixture migrate chromatographically along the nitrocellulose membrane in the card, if the test sample contains antiTreponema pallidum antibodies, the antibodies bind to the TP Ag 2 in the test region and a color develops indicating a positive result. No color development is an indication of negative results. As the mixture migrate to the control region the TP Ag 1 in the conjugate binds to the immobilized antibodies in the control region and a color develops. Color development in the control region is an indication that the TP Ag 1/colloidal gold conjugate is potent. Color development at the test and control region is an indication of valid positive results. Color development at the control region alone is an indication of valid negative results; however, color development at the test region alone indicates invalid test results.

\section{Statistical analysis}

Data analysis was performed using SPSS version 16.0. Difference in non-scalable variables such as age and sex of the study populations were assessed by Mann- 
Whitney $\mathrm{U}$ rank sum with $p$ values $<0.05$ considered significant ( $\mathrm{Cl}$ : 95\%). Chi-square $\left(\chi^{2}\right)$ test was used to determine associations between categorical variables. $p$ values $<0.05$ was considered statistically significant. Univariate analysis was used to predict the association between HIV, HBV, HCV and Syphilis infections among people with psychiatric disorders among the study population and risk factors.

\section{Results}

\section{Participant characteristics}

A total of two hundred (200) psychiatric patients (Mean age ( $35.60 \pm 12.06$ years) admitted at the Ankaful psychiatric hospital participated in this study. They comprised of $55 \%$ males $\{110 / 200$ (mean age $33.3 \pm 10.9$

Table 1: General characteristics of study participants.

\begin{tabular}{|c|c|c|c|c|}
\hline \multirow[t]{2}{*}{ Characteristics } & Male & Female & Total & $P$-value \\
\hline & $(n=110)$ & $(n=90)$ & $(n=200)$ & \\
\hline Mean Age \pm SD (years) & $33.28 \pm 10.88$ & $38.43 \pm 12.87$ & $35.60 \pm 12.06$ & \\
\hline \multicolumn{5}{|l|}{ Age group $\mathrm{n}(\%)$} \\
\hline$<20$ & $5(55.6)$ & $4(44.4)$ & $9(4.5)$ & \multirow[t]{6}{*}{0.083} \\
\hline $20-29$ & $45(67.2)$ & $22(32.8)$ & $67(33.5)$ & \\
\hline $30-39$ & $34(54.8)$ & $28(45.2)$ & $62(31.0)$ & \\
\hline $40-49$ & $15(48.4)$ & $16(51.6)$ & $31(15.5)$ & \\
\hline $50-59$ & $7(31.8)$ & $15(68.2)$ & $22(11.0)$ & \\
\hline$\geq 60$ & $4(44.4)$ & $5(55.6)$ & $9(4.5)$ & \\
\hline \multicolumn{5}{|l|}{ Marital status } \\
\hline Single & $80(65.6)$ & $42(34.4)$ & $122(61.0)$ & \multirow[t]{4}{*}{0.001} \\
\hline Married & $25(41.0)$ & $36(59.0)$ & $61(30.5)$ & \\
\hline Divorced & $4(44.4)$ & $5(55.6)$ & $9(4.5)$ & \\
\hline Widowed & $1(12.5)$ & $7(87.5)$ & $8(4.0)$ & \\
\hline \multicolumn{5}{|l|}{ Educational Level } \\
\hline None & $6(28.6)$ & $15(71.4)$ & $21(10.5)$ & \multirow[t]{4}{*}{0.005} \\
\hline Basic & $36(47.4)$ & $40(52.6)$ & $76(38.0)$ & \\
\hline Secondary & $42(64.6)$ & $23(35.4)$ & $65(32.5)$ & \\
\hline Tertiary & $26(68.4)$ & $12(31.6)$ & $38(19.0)$ & \\
\hline \multicolumn{5}{|l|}{ Employment } \\
\hline Yes & $58(52.3)$ & $53(47.7)$ & $111(55.5)$ & \multirow[t]{2}{*}{0.383} \\
\hline No & $52(58.4)$ & $37(41.6)$ & $89(44.5)$ & \\
\hline \multicolumn{5}{|l|}{ Number of children } \\
\hline None & 70 (72.9) & $26(27.1)$ & $96(48.0)$ & \multirow[t]{3}{*}{$<0.0001$} \\
\hline 3-Jan & $32(47.8)$ & $35(52.2)$ & $67(33.5)$ & \\
\hline 6-Apr & $8(25.0)$ & $24(75.0)$ & $32(16.0)$ & \\
\hline$>6$ & $0(0.0)$ & $5(100)$ & $5(2.5)$ & \\
\hline
\end{tabular}

Table 2: Sero-prevalence of HIV, HBV, HCV and Syphilis of participants categorized by gender and age.

\begin{tabular}{|l|l|l|l|l|}
\hline Characteristics & $\begin{array}{l}\text { HIV-1/2 antibody } \\
\text { positive (N= 10; N \%) }\end{array}$ & $\begin{array}{l}\text { HBV surface antigen } \\
\text { positive (N = 19; N \%) }\end{array}$ & $\begin{array}{l}\text { HCV antibody } \\
\text { positive (N = 4; N \%) }\end{array}$ & $\begin{array}{l}\text { Treponema pallidum antibody } \\
\text { positive (N = 27; N \%) }\end{array}$ \\
\hline Gender & $3(30.0)$ & $10(52.6)$ & $3(75.0)$ & $12(44.4)$ \\
\hline Male & $7(70.0)$ & $9(47.4)$ & $1(25.0)$ & $15(55.6)$ \\
\hline Female & & & \\
\hline Age group n (\%) & $0(0.0)$ & $0(0.0)$ & $0(0.0)$ & $1(3.7)$ \\
\hline$<20$ & $3(30.0)$ & $7(36.8)$ & $2(50.0)$ & $6(22.2)$ \\
\hline $20-29$ & $4(40.0)$ & $9(47.4)$ & $1(25.0)$ & $11(40.7)$ \\
\hline $30-39$ & $2(20.0)$ & $2(10.5)$ & $0(0.0)$ & $4(14.8)$ \\
\hline $40-49$ & $1(10.0)$ & $1(5.3)$ & $1(25.0)$ & $3(11.1)$ \\
\hline $50-59$ & $0(0.0)$ & $0(0.0)$ & $0(0.0)$ & $2(7.4)$ \\
\hline$\geq 60$ & &
\end{tabular}


years) $\}$ and $45 \%$ females $\{90 / 200$ (mean age $38.4 \pm 12.9$ years). Factors such as marital status, educational level and number of children showed significant differences whiles age and employment status showed no significant differences. Results of general characteristics of participants is presented in Table 1.

\section{Sero-prevalence of HIV, HBV, HCV and syphilis}

The overall sero-prevalence for HIV was $5 \%$ $(10 / 200)$ out of which $70 \%(7 / 10)$ were females with the remaining $30 \%(3 / 10)$ being males (Table 2). Seroprevalence for HBV was $9.5 \%$ (19/200) (comprising $52.6 \%(10 / 19)$ males and $47.4 \%(9 / 19)$ females) and the total prevalence for HCV was $2 \%$ (4/200). Overall seroprevalence for syphilis among study participants was $13.5 \%(27 / 200)$ which comprised of $44.4 \%$ male $(12 / 27)$ and $55.6 \%$ female (15/27). Results are presented in Table 2.

Co-infections and multiple infections among study participants showed $1.5 \%$ (3/200) sero-prevalence of HBV and HIV co-infections (Figure 1). HBV and syphilis co-infections was $1.0 \%(2 / 200)$ and HIV and Syphilis co-infection was $0.5 \%(1 / 200)$. HCV and syphilis coinfection was also $0.5 \%(1 / 200)$. Prevalence of patients who had HIV, HBV and Syphilis was 0.5\% (1/200).

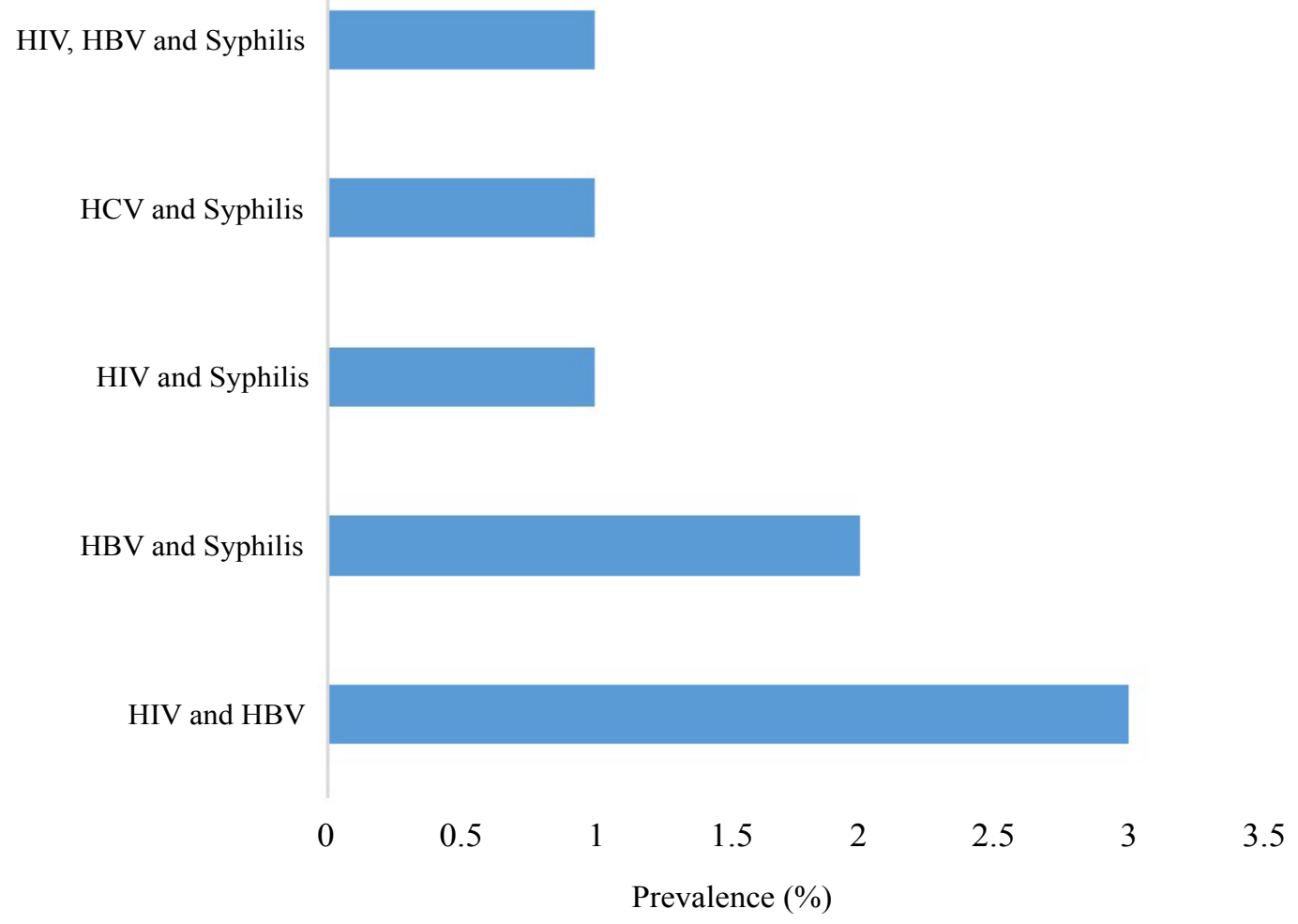

Figure 1: Prevalence of co-infections and multiple infections among study participants.

Table 3: Psychiatric diagnosis associated with HIV, HBV, HCV and Syphilis sero-positivity in participants by univariate analysis.

\begin{tabular}{|c|c|c|c|c|c|c|c|c|c|c|c|c|}
\hline \multirow[t]{2}{*}{$\begin{array}{l}\text { Psychiatric } \\
\text { Diagnosis }\end{array}$} & \multicolumn{3}{|c|}{$\begin{array}{l}\text { HIV-1/2 antibody } \\
\text { positive }\end{array}$} & \multicolumn{3}{|c|}{$\begin{array}{l}\text { HBV surface antigen } \\
\text { positive }\end{array}$} & \multicolumn{3}{|c|}{ HCV antibody positive } & \multicolumn{3}{|c|}{$\begin{array}{l}\text { Treponema pallidum } \\
\text { antibody positive }\end{array}$} \\
\hline & $\begin{array}{l}\mathrm{n} / \mathrm{N} \% \\
(\mathrm{~N}=10)\end{array}$ & $x^{2}$ & $\begin{array}{l}P \\
\text { value }\end{array}$ & $\begin{array}{l}\mathrm{n} / \mathrm{N} \% \\
(\mathrm{~N}=19)\end{array}$ & $x^{2}$ & $P$ value & $\begin{array}{l}n / N \% \\
(N=4)\end{array}$ & $x^{2}$ & $\mathbf{P}$ value & $\begin{array}{l}\mathrm{n} / \mathrm{N} \% \\
(\mathrm{~N}=27)\end{array}$ & $x^{2}$ & $\begin{array}{l}P \\
\text { value }\end{array}$ \\
\hline $\begin{array}{l}\text { Psychotic } \\
\text { disorder }\end{array}$ & - & - & - & $1(5.3)$ & 3.13 & 0.17 & $1(25)$ & 0.017 & 0.089 & $5(18.5)$ & 9.45 & $0.04^{*}$ \\
\hline Bipolar & $1(10)$ & 1.33 & 0.24 & $4(21.1)$ & 0.02 & 0.81 & - & - & - & $5(18.5)$ & 9.45 & $0.04^{*}$ \\
\hline Substance abuse & - & - & - & $3(15.7)$ & 0.35 & 0.78 & $1(25)$ & 0.017 & 0.089 & $1(3.7)$ & 0.15 & 0.81 \\
\hline Seizure disorders & $1(10)$ & 1.33 & 0.24 & $3(15.7)$ & 0.35 & 0.35 & - & - & - & $1(3.7)$ & 0.15 & 0.81 \\
\hline Schizophrenia & $6(60)$ & 5.3467 & $0.02^{*}$ & $8(42.1)$ & 8.51 & $0.04^{*}$ & $2(25)$ & 0.93 & 0.33 & $8(29.6)$ & 11.25 & $0.03^{*}$ \\
\hline Depression & $2(20)$ & 0.197 & 0.65 & - & - & - & - & - & - & $5(18.5)$ & 9.45 & $0.04^{*}$ \\
\hline Others & - & - & - & - & - & - & - & - & - & $2(7.4)$ & 0.35 & 0.7 \\
\hline
\end{tabular}

$p$ value was calculated by Pearson's Chi square $\left(X^{2}\right)$ with confidence interval $(\mathrm{Cl})$ of $95 \%$ and 1 degree of freedom (df). $p$ values less than 0.05 were considered statistically significant and are indicated in bold fonts $\left(^{*}\right)$. 


\section{Test of association between HBV, HIV, HCV and} syphilis sero-positivity and psychotic diagnosis

Psychotic diagnosis of the participants was related to sero-positivity of HIV, HBV, HCV and syphilis by univariate analysis. Results indicated a significant association between Schizophrenia diagnosis the seropositivity for anti-HIV antibodies, anti-HBV antibodies and anti-T. pallidum antibodies (Table 3). Additionally, the results also indicated a significant association between psychotic disorder, bipolar and depression diagnosis association with anti- T. pallidum antibodies. There was however no significant association between $\mathrm{HCV}$ sero-positivity and psychotic diagnosis.

Test of association of sexual lifestyles with HIV, HBV, HCV and syphilis sero-positivity in participants by univariate analysis

To understand the association of sexual lifestyles and sero-positivity of HIV, HBV, HCV and syphilis, we performed a univariate analysis. Results obtained indicated that heterosexuality was significantly associated with HIV, HBV, HCV and syphilis seropositivity (Table 4). In addition, we found out that participants who had regular sex partners were significantly associated with HIV infections. Majority of the study subjects who tested positive for HBV, HCV and syphilis had no history of forced sexual activity such as exchange of sex for money, food, shelter or drugs. However, there was no significant difference in terms of condom usage and infections with HIV, HBV, HCV and syphilis although a greater number of participants admitted to the use of condoms during sex (Table 4).

\section{Discussion}

In this study, we show the prevalence of HIV, HCV, $\mathrm{HBV}$ and syphilis infections in patients with psychiatric disorders. This is the first published study of its kind in a psychiatric hospital in Ghana.

We found a trend towards a higher HIV-prevalence among this population, with a prevalence of $5 \%$, which was significantly higher than the national prevalence of $1.6 \%$ [13]. Psychotic symptoms in HIV positive patients have been associated to the direct neuropathic effect of the virus on the brain or may be secondary due to the effect of medication or substance abuse, secondary due to opportunistic infections or from the psychological impact of the knowledge of an HIV diagnosis [14]. There are reports that suggest that mental illness is a risk factor for developing HIV infection [15-18]. Psychiatric patients may show high-risk sexual behaviour, suffer less often from social exclusion that may lead to exchange of sex for money or goods, and have less severe cognitive deficit that impairs judgement or the ability to negotiate safe sex [19]. It is well established that drugs of abuse alter judgment, induce loss-of-inhibition, and play a massive role in the spread of HIV by leading to engagement in impulsive and unsafe behaviors [20].

In Ghana, available evidence shows that viral hepatitis is ten times more common than human immune deficiency virus (HIV) infection [11]. However, there is no comprehensive viral hepatitis prevention and control programme analogous to that of HIV/AIDS that provides public awareness of the disease and access to treatment. Previous studies on Ghanaian blood donors show HBV prevalence at $>10 \%$, increasing in the rural

Table 4: Association of sexual lifestyles with HIV, HBV, HCV and Syphilis sero-positivity in participants by univariate analysis.

\begin{tabular}{|c|c|c|c|c|c|c|c|c|c|c|c|c|}
\hline & \multicolumn{3}{|c|}{$\begin{array}{l}\text { HIV-1/2 antibody } \\
\text { positive }\end{array}$} & \multicolumn{3}{|c|}{$\begin{array}{l}\text { HBV surface antigen } \\
\text { positive }\end{array}$} & \multicolumn{3}{|c|}{ HCV antibody positive } & \multicolumn{3}{|c|}{$\begin{array}{l}\text { Treponema pallidum } \\
\text { antibody positive }\end{array}$} \\
\hline & $\begin{array}{l}n / N= \\
(N=10)\end{array}$ & $x^{2}$ & $\begin{array}{l}P \\
\text { value }\end{array}$ & $\begin{array}{l}\mathrm{n} / \mathrm{N} \% \\
(\mathrm{~N}=19)\end{array}$ & $x^{2}$ & $P$ value & $\begin{array}{l}\mathrm{n} / \mathrm{N} \% \\
(\mathrm{~N}=4)\end{array}$ & $x^{2}$ & $P$ value & $\begin{array}{l}\mathrm{n} / \mathrm{N} \% \\
(\mathrm{~N}=27)\end{array}$ & $x^{2}$ & $P$ value \\
\hline \multicolumn{13}{|c|}{ Sexual Orientation } \\
\hline Heterosexual & $10(100)$ & 0.36 & 0.009 & $18(94.7)$ & 15.23 & $<0.0001$ & $4(100)$ & 6.28 & 0.004 & $27(100)$ & 11.25 & 0.03 \\
\hline Homosexual & - & - & - & $1(5.3)$ & - & 0.25 & - & - & - & - & - & - \\
\hline Bisexual & - & - & - & - & - & - & - & - & - & - & - & - \\
\hline \multicolumn{13}{|c|}{ Regular sexual partner(s) } \\
\hline Yes & $9(90.0)$ & 12.12 & 0.013 & $10(52.6)$ & 5.64 & 0.304 & $1(25.0)$ & 0.37 & 0.509 & $10(37.0)$ & 0.23 & 0.55 \\
\hline No & $1(10.0)$ & 0.15 & 0.23 & $9(47.4)$ & 4.56 & 0.25 & $3(75.0)$ & 0.71 & 0.48 & $17(63.0)$ & 0.16 & 0.66 \\
\hline \multicolumn{13}{|c|}{ History of forced sex? } \\
\hline Yes & - & - & - & $1(5.3)$ & 0.36 & 0.239 & $1(25.0)$ & 0.35 & 0.578 & $7(25.9)$ & 0.23 & 0.094 \\
\hline No & $10(100)$ & 2.59 & 0.01 & $18(94.7)$ & 9.56 & 0.02 & $3(75.0)$ & 5.64 & 0.06 & $20(74.1)$ & 6.32 & 0.03 \\
\hline \multicolumn{13}{|c|}{ Have you ever used condoms during sex? } \\
\hline Yes & $5(50.0)$ & 2.36 & 0.845 & $8(42.1)$ & 0.59 & 0.654 & $2(50.0)$ & 0.11 & 0.903 & $13(48.1)$ & 0.65 & 0.898 \\
\hline No & $5(50.0)$ & 2.36 & 0.845 & $11(57.9)$ & 0.98 & 0.95 & $2(50.0)$ & 0.67 & 0.74 & $14(51.9)$ & 0.87 & 0.59 \\
\hline
\end{tabular}

$p$ value was calculated by Pearson's Chi square $\left(X^{2}\right)$ with confidence interval $(\mathrm{Cl})$ of $95 \%$ and 1 degree of freedom (df). $p$ values less than 0.05 were considered statistically significant and are indicated in bold fonts $\left({ }^{*}\right)$. 
and deprived areas [21,22], while that of HCV was estimated at more than $3 \%$ in some rural communities [23].

Additionally, we analyzed the related risk factors associated with blood-borne viruses and syphilis infections. Factors such as intravenous drug use and sexual behaviour, history of alcohol abuse, lack of condom use, having multiple sexual partners, sex trading, partner with a blood-borne virus were analyzed. There was no significant association between the bloodborne viruses, syphilis and forced sexual activity such as the exchange of sex for money, food, shelter or drugs. Consistent with our observation, majority of the participants were aware of the risk factors influencing the acquisition and spread of HIV, HBV, HCV and syphilis. The high level of awareness of blood borne viruses and syphilis infections exhibited by our study population could be associated with the vigorous HIV/ AIDS awareness campaign ongoing in Ghana [24].

Patients with severe mental illness who are HIV positive may have more than one psychiatric diagnosis, as well as an elevated prevalence of co-morbid medical disease such as TB and hypertension [25]. From this study, co-infection with other blood borne viruses and syphilis thus raises questions of possible extreme susceptibility of these patients. This therefore underscores the need for clinicians to be vigilant in detecting various neuropsychiatric manifestations of HIV infection. If detected timeously, co-morbidities addressed; and antiretroviral therapy and psychotropic initiated early, this would have a positive impact on the prognosis of a patient resulting in an improved quality of life. Staff at HIV treatment clinics should also have sufficient expertise in assessing the signs and symptoms of mental illness and realize the importance of referrals to specialists when necessary.

A possible study limitation, which merits discussion, is that participants were recruited from a treatment site and results may not be generalizable to all people with mental illness. However, we estimate that this limitation had minimal effects on our findings since most people consented, and the sample exceeded the calculated sample size.

\section{Recommendation and Conclusion}

From our study, we propose the need for routine HIV, HBV, HCV and syphilis screening over targeted, risk-based testing in psychiatric settings in Ghana. This routine screening in mental health settings will provide an opportunity for counselling and enhancing patients' knowledge of risk factors and prevention. Additionally, it will possibly identify many seropositive patients who may be overlooked in a system reliant on self-identified risk [26]. People with severe mental illness have higher all-cause mortality than those without [27]. It is possible that people with severe mental illness are not receiving recommended HIV, HCV, HBV and syphilis-related healthcare, since there is no regular testing of such infections.

In conclusion, mental health services in Ghana, which has a generalized HIV epidemic, need to take into account the frequent co-morbidity of HIV, viral hepatitis and syphilis. Periodic risk assessment, counselling and testing, and treatment and care of HIV, HCV, HBV and syphilis should be integrated with mental health services. Conversely, HIV treatment programs should take into account the high burden of mental illness among persons with HIV and integrate mental health assessments and interventions with routine HIV care. Our results could form the baseline data for decision making by the health authorities, as there is an urgent need for developing STI prevention and care strategies for this population in Ghana.

\section{References}

1. World Health Organization (2012) Risks to mental health: An overview of vulnerabilities and risk factors. Geneva, Switzerland.

2. Ibrahim A, Hor S, Bahar OS, Dwomoh D, McKay MM, et al. (2016) Pathways to psychiatric care for mental disorders: A retrospective study of patients seeking mental health services at a public psychiatric facility in Ghana. Int J Ment Health Syst 10: 63.

3. Azeem EM (2014) Treatment seeking behaviour of people living with mental illness in Regional Psychiatric hospitals in Ghana. University of Ghana.

4. Campos LN, Guimaraes MD, Carmo RA, Melo AP, Oliveira $\mathrm{HN}$, et al. (2008) HIV, syphilis, and hepatitis B and C prevalence among patients with mental illness: A review of the literature. Cad Saude Publica 4: S607-S620.

5. Mayston R, Kinyanda E, Chishinga N, Prince M, Patel V (2012) Mental disorder and the outcome of HIVIAIDS in low-income and middle-income countries: a systematic review. AIDS 2: S117-S135.

6. Bhugra D (2005) The global prevalence of schizophrenia. PLoS Med 2: e151.

7. Ofori-Atta A, Read UM, Lund C (2010) A situation analysis of mental health services and legislation in Ghana: Challenges for transformation. Afr J Psychiatry 13: 99-108.

8. Hughes E, Bassi S, Gilbody S, Bland M, Martin F (2016) Prevalence of HIV, hepatitis $B$, and hepatitis $C$ in people with severe mental illness: A systematic review and metaanalysis. Lancet Psychiatry 3: 40-48.

9. Lagios K, Deane FP (2007) Severe mental illness is a new risk marker for blood-borne viruses and sexually transmitted infections. Aust N Z J Public Health 31: 562-566.

10. Nkrumah B, Owusu M, Averu P (2011) Hepatitis B and C viral infections among blood donors. A retrospective study from a rural community of Ghana. BMC Res Notes 4: 529.

11. Dongdem JT, Kampo S, Soyiri IN, Asebga PN, Ziem JB, et al. (2012) Prevalence of hepatitis B virus infection among blood donors at the Tamale Teaching Hospital, Ghana (2009). BMC Res Notes 5: 115.

12. Rutterford C, Copas A, Eldridge S (2015) Methods for sample size determination in cluster randomized trials. Int $\mathrm{J}$ Epidemiol 44: 1051-1067. 
13. GAC (2017) Summary of the 2016 HIV Sentinel Survey Report. Ghana AIDS Commission, Ghana.

14. Meade CS, Sikkema KJ (2007) Psychiatric and psychosocial correlates of sexual risk behaviour among adults with severe mental illness. Community Ment Health J 43: 153169.

15. Angelino AF, Treisman GJ (2008) Issues in co-morbid severe mental illnesses in HIV infected individuals. Int Rev Psychiatry 20: 95-101.

16. Collins PY, Holman AR, Freeman MC, Patel V (2006) What is the relevance of mental health to HIVIAIDS care and treatment programs in developing countries? A systematic review. AIDS 20: 1571-1582.

17. Kelly JA, Murphy DA, Sikkema KJ, Somlai AM, Mulry GW, et al. (1995) Predictors of high and low levels of HIV risk behavior among adults with chronic mental illness. Psychiatr Serv 46: 813-818.

18. Senn TE, Carey MP (2009) HIV testing among individuals with a severe mental illness: Review, suggestions for research, and clinical implications. Psychol Med 39: 355363.

19. Joska JA, Kaliski SZ, Benatar SR (2008) Patients with severe mental illness: A new approach to testing for HIV. S Afr Med J 98: 213-217.

20. Ickovics JR, Meade CS (2002) Adherence to antiretroviral therapy among patients with HIV: A critical link between behavioral and biomedical sciences. J Acquir Immune Defic Syndr 31: S98-S102.
21. Agyeman AA, Ofori-Asenso R, Mprah A, Ashiagbor G (2016) Epidemiology of hepatitis $C$ virus in Ghana: A systematic review and meta-analysis. BMC Infect Dis 16: 391.

22. Nkrumah B, Owusu M, Frempong HO, Averu P (2011) Hepatitis $B$ and $C$ viral infections among blood donors from rural Ghana. Ghana Med J 45: 97-100.

23. Martinson FE, Weigle KA, Mushahwar IK, Weber JD, Royce $R$, et al. (1996) Seroepidemiological survey of hepatitis $B$ and $C$ virus infections in Ghanaian children. $J$ Med Virol 48: 278-283.

24. Amidu N, Owuredu WBKA, Addai-Mensah O, Alhassan A, Quaye L, et al. (2010) seroprevalence and risk factors for human immunodeficiency virus, Hepatitis B and $C$ Viruses infections among blood donors at the bolgatanga regional hospital in Bolgatanga, Ghana. Journal of the Ghana Science Association 12: 77-88.

25. Oppong Asante K, Oti-Boadi M (2013) HIVIAIDS knowledge among undergraduate university students: Implications for health education programs in Ghana. Afr Health Sci 13: 270-277.

26. Mutocheluh M, Owusu M, Kwofie TB, Akadigo T, Appau E, et al. (2014) Risk factors associated with hepatitis B exposure and the reliability of five rapid kits commonly used for screening blood donors in Ghana. BMC Res Notes 7: 873.

27. Prince M, Patel V, Saxena S, Mario Maj, Joanna Maselko, et al. (2007) No health without mental health. Lancet 370 : 859-877. 